\title{
The Effect of Fermentation Time (as Induced by Fermentation and Must Conditions) on the Chemical Profile and Quality of a Chenin blanc Wine
}

\author{
P. C. VAN ROOYEN AND A. TROMP \\ Oenological and Viticulture Research Institute (OVRI), Private Bag X5026, Stellenbosch, South Africa.
}

Submitted for publication: August 1982

Accepted for publication: September 1982.

\begin{abstract}
Pattern recognition and simple factorial analyses were applied to chemical profiles determined on wines resulting from an experiment in which the effect of grape solids, assimilable nitrogen, bentonite and fermentation temperature levels as well as culture aeration on total fermentation time was investigated. It was found that fermentation times of the untreated Chenin blanc must could be shortened considerably by increasing grape solid levels and/or nitrogen as well as temperature. However, it was established that the speeding up of fermentation at sub-optimal assimilable nitrogen levels leads to wines relatively high in higher alcohols and low in esters.
\end{abstract}

Several problems arise from stuck or lagging fermentations, amongst them space problems in cellars, marketing problems due to residual sugar, and a general belief that wine quality is also negatively affected. Several studies were conducted on this problem (Aries \& Kirsop, 1970; Agenbach, 1977; Groat \& Ough, 1978, Vos et al., 1979), resulting in the identification of several contributing factors, such as low must nitrogen and grape solids levels, low fermentation temperature and an inadequate supply of oxygen during the initial stages of fermentation. Agenbach (1977) defined a minimum level of $130 \mathrm{mg} / \ell$ must $\alpha$-amino nitrogen (expressed as lysine) for completion of fermentation; Aries \& Kirsop (1970) stressed the role of oxygen in ergosterol formation, while Groat \& Ough (1978) showed that $0,5 \%$ grape solids concentration was adequate to prevent lagging fermentation. Vos \& Gray (1980) demonstrated the utility of ammonium phosphate addition in nitrogen deficient musts in preventing hydrogen sulphide formation and lagging during fermentation.

The complex interactions of the various factors as outlined above with fermentation time, do not appear to have been studied in relation to wine quality and the production of flavour or other chemical compounds in wine. The purpose of this study was to investigate the effect of five must and fermentation treatments on total fermentation time, aroma composition of the wines and wine quality.

\section{MATERIALS AND METHODS}

Must treatments: Chenin blanc must from a vineyard known to be susceptible to lagging or stuck fermentations was subdivided into 64 duplicate fermentations in $20 \ell$ stainless steel containers. Using a factorial design, the following treatments were applied:

1. Four grape solids levels, namely 0,$0 ; 0,25 ; 0,5$ and 1,0 per cent obtained by adding different quantities of lees to filtered grape must.

2. Two $\alpha$-amino nitrogen levels of $130 \mathrm{mg} / \ell$ and $800 \mathrm{mg} /$ $\ell$ must, adjusted by the addition of ammonium phosphate.

3. Two fermentation temperatures, viz. $13^{\circ} \mathrm{C}$ and $20^{\circ} \mathrm{C}$.

4. Two levels of air application - no aeration and an aeration of 30 seconds 24 hours after inoculation by sparging through sintered glass at $10 \ell / \mathrm{min}$.
5. Two bentonite treatment levels, viz. no addition and an addition of $800 \mathrm{mg} / \ell$ prior to inoculation.

The yeast used was Saccharomyces cerevisiae (strain WE 14, OVRI collection), and a $5 \%$ inoculation of an actively fermenting anaerobic preculture was applied. Fermentation rates were recorded by daily mass determinations and periodic sugar analyses. At dryness the wines were filtered and cold stabilized $\left(0^{\circ} \mathrm{C}\right)$, while total fermentation time was also noted. After filtration and bottling the wines were stored at $0^{\circ} \mathrm{C}$ to prevent any changes in quality and/or composition. The wines were subsequently analysed for the compounds listed in Table 1. The individual volatile compounds were determined by the freon extraction - gaschromatographic method described by Marais \& Houtman (1979). Glycerol was determined by an enzymatic method (Junge, 1980), total polyphenols by the method of Singleton \& Rossi (1965), and colour by spectrophotometric measurement at $420 \mathrm{~nm}$ in $10 \mathrm{~mm}$ pathlength cuvettes. Total esters were determined by employing a distillation method, and total higher alcohols by the methods of the AOAC (1975).

TABLE 1

Independent variables quantified for 128 Chenin blanc wines

\begin{tabular}{clcl}
\hline NUMBER & VARIABLE & NUMBER & VARIABLE \\
\hline 1 & Glycerol & 11 & Hexyl acetate \\
2 & Acetaldehyde & 12 & Hexanol \\
3 & Colour & 13 & Ethyl octanoate \\
4 & Polyphenols & 14 & Ethyl decanoate \\
5 & Ethyl acetate & 15 & 2-Phenyl ethyl acetate \\
6 & Ethyl butyrate & 16 & Hexanoic acid \\
7 & i-Butanol & 17 & 2-Phenyl ethyl alcohol \\
8 & i-Amyl acetate & 18 & Octanoic acid \\
9 & i-Amyl alcohol & 19 & Total esters \\
10 & Ethyl hexanoate & 20 & Total higher alcohols \\
\hline
\end{tabular}

Data processing; A standard factorial analysis was performed using wine quality as dependent variable. Subsequently each of the 128 wines with its associated chemical data vector was analysed using the pattern recognition program "ARTHUR" (Harper et al., 1977). The patterns were studied in relation to total fermentation time (TFT) where supervised analyses were performed. TFT was used as a dependent variable in regression analysis, or as a 
basis for classifying the wines into different categories of total fermentation time. For the latter type of analysis (category analysis) the codes 1 to 4 were used for four categories of TFT as set out in Table 2. In the case of continuous property analyses, actual TFT values were used to weight variables according to correlation with the latter.

TABLE 2

Category classification according to total fermentation time (TFT)

\begin{tabular}{ccc}
\hline CATEGORY & TFT DAYS & NUMBER OF WINES \\
\hline 1 & 40 & 12 \\
2 & $21-40$ & 30 \\
3 & $13-20$ & 45 \\
4 & $0-12$ & 41 \\
\hline
\end{tabular}

The data were analysed using a batch of process version of the pattern recognition system "ARTHUR", executed on a UNIVAC 1110 computer of the University of Stellenbosch. The study was undertaken in two phases, the first being an initial study of the underlying data structure to postulate hypotheses regarding relationships amongst wine components and their relation to total fermentation time in order to reduce the larger number of variables. In the second phase selected pattern recognition analyses were performed on a reduced data set employing only those variables selected during the first part of the study. The following programmes were used:

\section{Programme function}

Calculate correlation and covariance matrices.

Perform a principal component analysis (PCA), and execute a Varimax rotation on the eigenvectors before a Karhuhen- Loeve transformaction and plotting of vector scores.
Feature selection creating orthogonal features based on their importance to classification. The criterion for importance in this case was correlation to property (total fermentation time).

Investigation of 380 variable ratios forimportance to classification by creating orthogonal features for plotting.

Calculate the interpattern distance matrix (in this case the

Mahanolobis distance) and use the nonlinear mapping technique for displaying the data.

Multidimensional multivariate regression computing a linear discriminant function maximizing multiple correlation.

All data were scaled to zero mean and a standard deviation of one before any of the above analyses were done. Finally PCA and non-linear mapping techniques were used to test the variable selection hypotheses and to construct a final visual display of the data structure.

\section{RESULTS AND DISCUSSION}

Raw data: To give an idea of magnitudes within the data set, a summary of the measurements is given in Table 3 .

Simple correlation coefficients: Of the correlation coefficients calculated amongst individual variables and fermentation time, only two were above 0,5 , viz. 0,62 for total polyphenols with fermentation time, and $-0,62$ for ethyl hexanoate, indicating a general increase in polyphenols and a general decrease in ethyl hexanoate with increasing total fermentation time.

TABLE 3

Basic statistics on raw data used for the study.

\begin{tabular}{lllll}
\hline Variable & $\begin{array}{l}\text { Mean } \\
(\mathrm{mg} / \ell)\end{array}$ & Minimum & Maximum & $\begin{array}{l}\text { Standard } \\
\text { deviation }\end{array}$ \\
\hline Glycerol & $4,709 \mathrm{E}+002$ & $2,900 \mathrm{E}+002$ & $7,600 \mathrm{E}+002$ & $7,368 \mathrm{E}+001$ \\
Acetaldehyde & $5,243 \mathrm{E}+001$ & $4,100 \mathrm{E}+001$ & $7,000 \mathrm{E}+001$ & $7,067 \mathrm{E}+000$ \\
Colour* & $5,084 \mathrm{E}+001$ & $3,400 \mathrm{E}+001$ & $8,900 \mathrm{E}+001$ & $1,129 \mathrm{E}+001$ \\
Polyphenols & $2,123 \mathrm{E}+002$ & $1,520 \mathrm{E}+002$ & $2,900 \mathrm{E}+002$ & $1,995 \mathrm{E}+001$ \\
Ethyl acetate & $3,304 \mathrm{E}+001$ & $2,310 \mathrm{E}+001$ & $4,560 \mathrm{E}+001$ & $5,683 \mathrm{E}+000$ \\
Ethyl butyrate & $4,387 \mathrm{E}-001$ & $2,500 \mathrm{E}-001$ & $7,400 \mathrm{E}-001$ & $1,438 \mathrm{E}-001$ \\
i-Butanol & $1,469 \mathrm{E}+001$ & $8,100 \mathrm{E}+000$ & $2,460 \mathrm{E}+001$ & $3,520 \mathrm{E}+000$ \\
i-Amyl acetate & $3,921 \mathrm{E}+000$ & $2,440 \mathrm{E}+000$ & $5,380 \mathrm{E}+000$ & $8,561 \mathrm{E}-001$ \\
i-Amyl alcohol & $1,407 \mathrm{E}+002$ & $8,520 \mathrm{E}+001$ & $2,445 \mathrm{E}+002$ & $3,979 \mathrm{E}+001$ \\
Ethyl hexanoate & $1,006 \mathrm{E}+000$ & $6,150 \mathrm{E}-001$ & $1,371 \mathrm{E}+000$ & $1,829 \mathrm{E}-001$ \\
Hexyl acetate & $2,440 \mathrm{E}-001$ & $1,100 \mathrm{E}-001$ & $3,900 \mathrm{E}-001$ & $6,791 \mathrm{E}-002$ \\
Hexanol & $1,300 \mathrm{E}+000$ & $6,000 \mathrm{E}-002$ & $2,030 \mathrm{E}+000$ & $2,389 \mathrm{E}-001$ \\
Ethyl octanoate & $1,358 \mathrm{E}+000$ & $9,800 \mathrm{E}-001$ & $1,670 \mathrm{E}+000$ & $1,339 \mathrm{E}-001$ \\
Ethyl decanoate & $7,061 \mathrm{E}-001$ & $3,600 \mathrm{E}-001$ & $1,060 \mathrm{E}+000$ & $1,211 \mathrm{E}-001$ \\
2-Phenylethyl acetate & $2,088 \mathrm{E}-001$ & $1,200 \mathrm{E}-001$ & $2,900 \mathrm{E}-001$ & $3,863 \mathrm{E}-002$ \\
Hexanoic acid & $5,905 \mathrm{E}+000$ & $4,120 \mathrm{E}+000$ & $7,890 \mathrm{E}+001$ & $1,050 \mathrm{E}+000$ \\
2-Phenyl ethyl alcohol & $1,370 \mathrm{E}+001$ & $6,000 \mathrm{E}+000$ & $2,635 \mathrm{E}+001$ & $6,362 \mathrm{E}+000$ \\
Octanoic acid & $7,612 \mathrm{E}+000$ & $5,690 \mathrm{E}+000$ & $9,420 \mathrm{E}+000$ & $9,201 \mathrm{E}+001$ \\
Total esters & $4,017 \mathrm{E}+001$ & $2,890 \mathrm{E}+001$ & $2,432 \mathrm{E}+001$ & $6,787 \mathrm{E}+000$ \\
Total higher alcohols & $1,708 \mathrm{E}+002$ & $1,038 \mathrm{E}+002$ & $2,911 \mathrm{E}+002$ & $4,857 \mathrm{E}+001$ \\
\hline
\end{tabular}

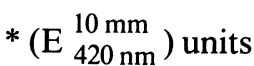


Principal component analysis: The preliminary analysis on the scaled data after applying the Varimax rotation to the eigenvectors resulted in the first three principal compoments explaining $82,5 \%$ of the total variation. The relevant data including factor loadings are given in Table 4.

TABLE 4

Factor loadings for the first three eigenvectors after rotation

\begin{tabular}{l|r|r|r}
\hline Variable & $\begin{array}{r}\text { Factor I } \\
56,8 \%\end{array}$ & $\begin{array}{c}\text { Factor II } \\
16,3 \%\end{array}$ & $\begin{array}{c}\text { Factor III } \\
9,4 \%\end{array}$ \\
\hline Glycerol & 0,14 & 0,04 & 0,09 \\
Acetaldehyde & $-0,04$ & $-0,35$ & 0,32 \\
Colour & 0,03 & $-0,05$ & 0,12 \\
Total polyphenols & $-0,26$ & 0,03 & 0,26 \\
Ethyl acetate & 0,27 & $-0,18$ & 0,12 \\
Ethyl butyrate & 0,33 & $-0,14$ & 0,12 \\
i-Butanol & $-0,08$ & 0,38 & 0,08 \\
i-Amyl acetate & 0,33 & $-0,13$ & $-0,07$ \\
i-Amyl alcohol & $-0,12$ & 0,38 & $-0,08$ \\
Ethyl hexanoate & 0,35 & $-0,04$ & 0,06 \\
N-hexyl acetate & 0,17 & $-0,35$ & $-0,08$ \\
Hexanol & $-0,10$ & 0,10 & 0,20 \\
Ethyl octanoate & 0,33 & $-0,03$ & $-0,10$ \\
Ethyl decanoate & $-0,03$ & 0,05 & $-0,12$ \\
2-Phenethyl acetate & 0,00 & 0,10 & $-0,77$ \\
Hexanoic acid & 0,33 & $-0,17$ & 0,05 \\
2-Phenethyl alcohol & $-0,19$ & 0,33 & $-0,25$ \\
Octanoic acid & 0,29 & $-0,22$ & $-0,04$ \\
Total esters & 0,29 & $-0,17$ & 0,10 \\
Total higher alcohols & $-0,13$ & 0,39 & $-0,10$ \\
\hline & \multicolumn{3}{|c}{} \\
\hline
\end{tabular}

Factor I may be described as being mainly indicative of fruitiness due to relatively high loadings for ethyl butyrate, i-amyl acetate, ethyl hexanoate, ethyl octanoate and hexanoic acid. Higher alcohols characterize factor II to a large extent, while 2 phenethyl acetate plays the major role in the case of factor III. A plot of total fermentation time against factor I demonstrates the general trend in terms of ester formation for the wines. Positive values on the $\mathrm{x}$-axis increase towards higher ester concentrations, whereas wine on the negative side of the central axis tend towards lower ester concentrations (Fig. 1). In interpreting the figures it must be noted that the various symbols depicting individual treatments are in some case superimposed. General trends which can be identified from the latter scatter diagram on which treatments have been decoded as far as possible, are:

- without the application of nitrogen the promotion of faster fermentation by fermenting at higher temperature or higher levels of solids, does not lead to higher ester concentrations (compare wines coded with and without open circles).

- the negative effects of low grape solids contents cannot be overcome by higher fermentation temperatures, aeration of the must or the addition of bentonite, both in terms of faster fermentation and ester formation.

- the addition of nitrogen to low turbidity musts leads to faster fermentation times, but in general not to high ester concentrations.

- high turbidity musts need adequate levels of nitrogen for high ester formation.

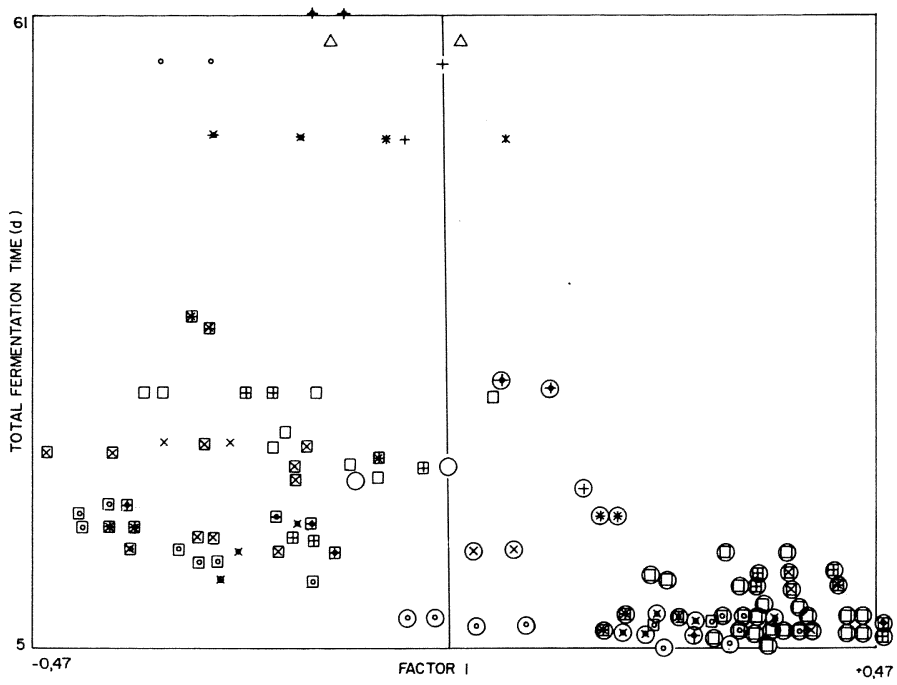

Fig. 1

Plot of principal component factor scores (Factor 1) against total fermentation time.

$\Delta:$ control samples

0 : high $\alpha$-amino nitrogen level $(800 \mathrm{mg} / \ell)$

$\square$ : samples with added grape solids

$\mathrm{X}$ : aerated musts

${ }^{\circ}$ : fermented at $20^{\circ} \mathrm{C}$

+ : bentonite addition

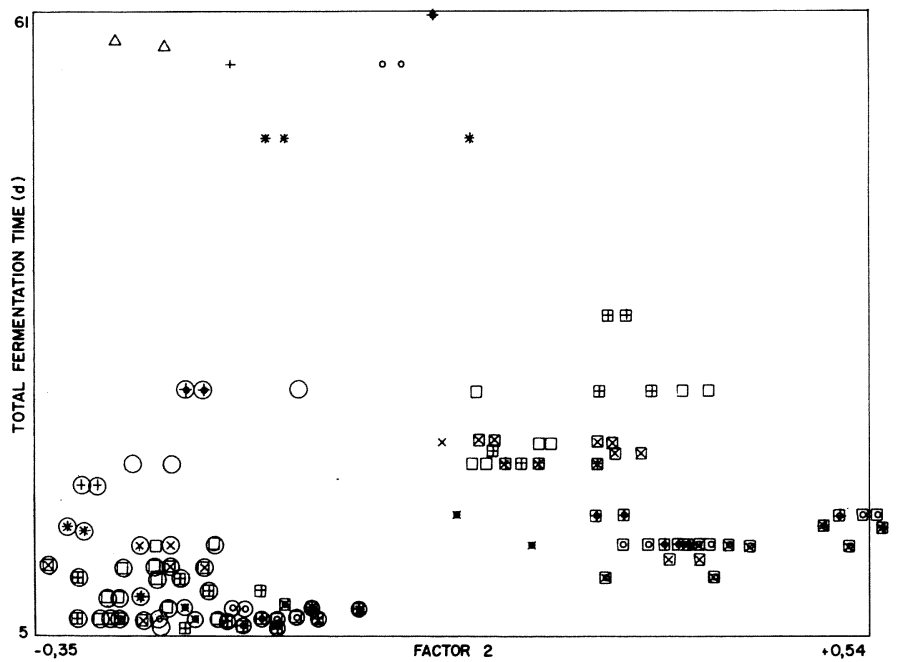

Fig. 2

Plot of principal component factor scores (Factor 2) against total fermentation time.

$\Delta:$ control samples

0 : high $\alpha$-amino nitrogen level $(800 \mathrm{mg} / \ell)$

$\square$ : samples with added grape solids

$\mathrm{X}$ : aerated musts

$\circ$ : fermented at $20^{\circ} \mathrm{C}$

+ : bentonite addition

The scatter diagram representing a plot of factor II scores against fermentation time is given in Fig. 2. Again treatments have been decoded as far as overprinting by the computer allowed. Interesting facts emerged from the latter figure:

- speeding up fermentation by higher fermentation temperatures and/or high grape solids leads to increased higher alcohol formation when $\alpha$-amino nitrogen levels are sub-optimal. 
- in the case of completely clear musts, increase of temperature or must aearation does not alleviate the lagging fermentation problem. The addition of nitrogen without grape solids leads to intermediate fermentation times and low higher alchohol formation.

- at adequate grape solids and nitrogen levels, increases in fermentation temperature increase higher alcohol formation above the levels of wines fermented at lower temperature.

Thus at low fermentation temperatures moderate levels of grape solids (around 0,25\%), with adjustment of assimilable nitrogen seem to be conducive to high ester concentration, low higher alcohol formation and "normal" fermentation times. Fermentation times as short as seven days still conform to this reasoning. Data from a simple factorial analysis employing overall and aroma quality as dependent variables confirm this deduction on a global quality basis (Table 5).
DISTANCE used to calculate a distance matrix according to the Mahanolobis method (Harper, et al., 1977). To display the results a non linear mapping technique was used to determine whether certain feature profiles differed from others. Each wine was again coded according to total fermentation time. From the results presented in Fig. 4 a successful mapping of the four fermentation time classes is seen, confirming basically different feature profiles for the four classes, with minimum overlapping. An interesting result is that wines resulting from stuck fermentations were split into two groups, viz. region $\mathrm{A}$ for wines fermented at $12^{\circ} \mathrm{C}$ and region $\mathrm{B}$ for wines fermented at $20^{\circ} \mathrm{C}$. The relatively large distance between these two groups signify widely diverging chemical profiles.

Analyses on categorized data: To produce more contrasting categories for the supervised analyses, the categories were redifined as follows: category 1: total fermentation time of more than 40 days; category 2: 21 - 40 days; category 3: 11 - 20 days and category 4 : less than 10 days. Using a

TABLE 5

Composite means for total fermentation time (TFT), overall wine quality (\%), aroma quality (\%) as affected by grape solids, nitrogen, aeration, bentonite addition and temperature levels of a Chenin blanc must.

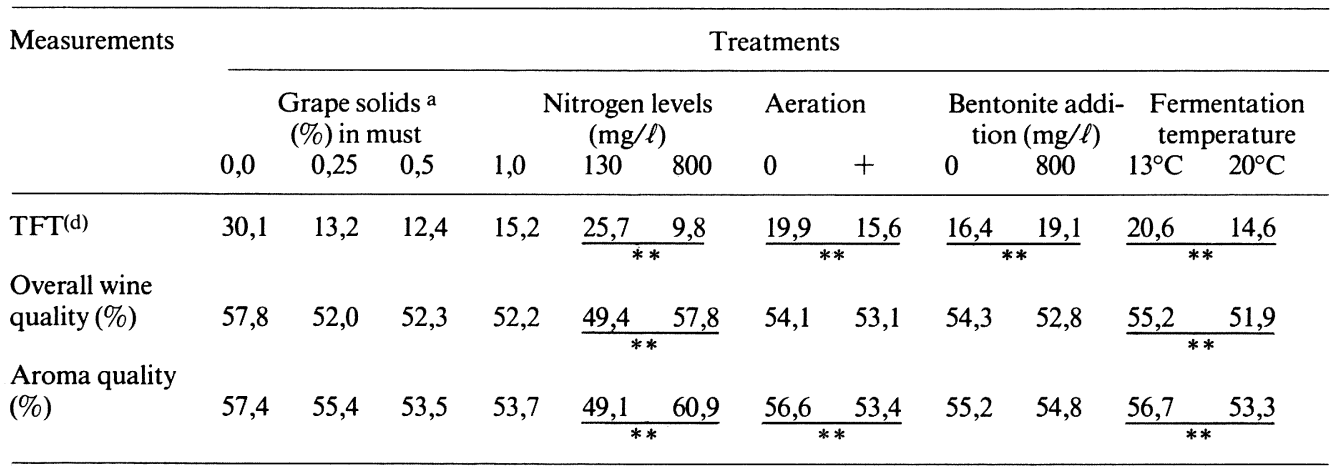

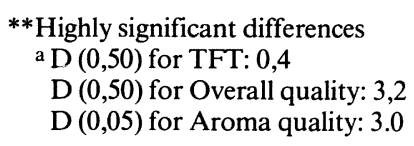

Variable selection: The selection was done according to PCA principles employing correlation to fermentation time weighting: Ten orthogonal features were selected employing the above method, and comprise the list as set out in Table 6. Ethyl hexanoate apparently constitutes the most important variable, followed by i-butanol. A plot of the orthonormalized features of the two variables is given in Fig. 3, indicating tendencies for lower i-butanol concentrations at long fermentation times, and high ethyl hexanoate concentrations at short fermentation times. These distributions could not be clearly connected to must treatments as in the previous section.

No clear relationships were found between TFT and other variables. Of 380 variable ratios only one was significant, namely the total polyphenols/ethyl hexanoate ratio. However, the significance of the latter ratio was mostly due to a decrease of ethyl hexanoate with total fermentation time, as shown in Fig. 3.

Cluster analysis: Employing the same technique as described above, the programme SELECT was used to select 9 original as well as 7 variable ratios. These two variable sets were subsequently combined, and the programme multidimensional regression method to compute linear discriminant functions for each category, a $90,8 \%$ correct classification into the four categories was obtained, with weight vectors as set out in Table 7 . For category 1 heavily weighted features were colour, hexanol, hexyl acetate, hexanoic acid and iso-amyl alcohol, while category 2 was dominated by positively weighted iso-amyl

TABLE 6

Orthogonal features selected on basis of correlation to fermentation time weighting.

\begin{tabular}{ll}
\hline Variable & Relative Weight \\
\hline Ethyl hexanoate & 0,39 \\
i-Butanol & 0,11 \\
Polyphenols & 0,10 \\
2-phenyl ethyl acetate & 0,05 \\
Hexanoic acid & 0,03 \\
Ethyl butyrate & 0,04 \\
Hexyl acetate & 0,02 \\
Colour & 0,02 \\
i-Amyl acetate & 0,01 \\
Ethyl octanoate & 0,00 \\
\hline
\end{tabular}




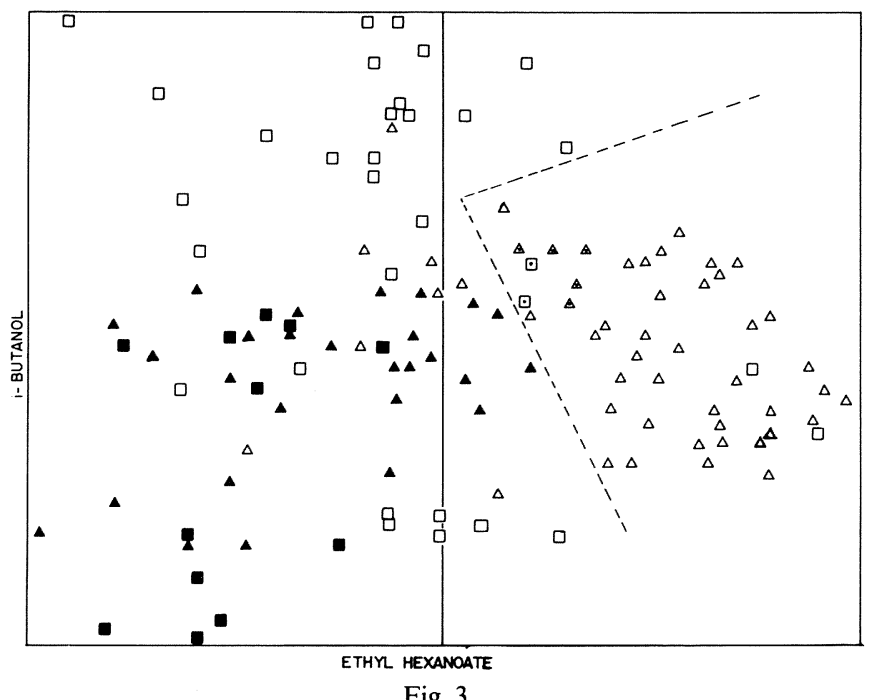

Fig. 3.

Plot of selected, orthogonal features using correlation to total fermentation time (TFT) weighting.

$\triangle: 0-12$ days TFT

口: $13-20$ days TFI

A : 21 - 40 days TFT

- : $40+$ days TFT

alcohol and negatively weighted acetaldehyde. It seems that lagging fermentations induced by clear musts lead to more intense colour and higher hexyl acetate concentrations. Fast fermentations induced by grape solids, nitrogen and to a lesser extent temperature, air and bentonite additions lead to wines high in 2-phenyl ethyl acetate. It is interesting to note that acetaldehyde and total higher alcohols were most heavily weighted in category 3 (11 $20 \mathrm{~d})$. The same observation holds for hexanol, whereas a relatively large negative weight was found for 2-phenyl ethyl acetate.

TABLE 7

Weight vectors for discriminant functions categorising the data into four fermentation time classes.

\begin{tabular}{l|r|r|r|r}
\hline Variable & $\begin{array}{c}\text { Category } 1 \\
<40 \mathrm{~d}\end{array}$ & $\begin{array}{c}\text { Category } 2 \\
21-40 \mathrm{~d}\end{array}$ & $\begin{array}{c}\text { Category } 3 \\
11-20 \mathrm{~d}\end{array}$ & $\begin{array}{c}\text { Category } 4 \\
<10 \mathrm{~d}\end{array}$ \\
\hline 2 Phenyl ethyl alcohol & 0,35 & $-0,55$ & $-1,57$ & 1,76 \\
Glycerol & 0,57 & $-0,54$ & 0,61 & $-0,64$ \\
Ethyl decanoate & 1,27 & 0,14 & $-0,34$ & $-1,06$ \\
Colour & 3,38 & $-1,70$ & 0,38 & $-2,06$ \\
Hexanol & $-2,81$ & $-3,63$ & 8,25 & $-1,81$ \\
Polyphenols & $-0,95$ & $-3,97$ & 2,46 & 2,46 \\
2-Phenyl ethyl acetate & 0,73 & 3,58 & $-8,76$ & 4,46 \\
Ethyl hexanoate & $-0,14$ & $-2,42$ & 2,21 & 0,35 \\
i-Butanol & $-1,03$ & 0,14 & 0,18 & 0,71 \\
Acetaldehyde & $-0,93$ & $-5,14$ & 10,03 & $-3,96$ \\
Ethyl octanoate & $-0,35$ & 1,09 & $-0,09$ & $-0,65$ \\
Ethyl acetate & $-0,49$ & 1,54 & $-2,34$ & 1,29 \\
Octanoic acid & 0,15 & $-0,20$ & $-0,05$ & 0,10 \\
Ethyl butyrate & 1,84 & $-2,63$ & 0,55 & 0,25 \\
Hexyl acetate & 4,16 & $-2,22$ & $-0,75$ & $-1,19$ \\
i-Amylalcohol & $-3,07$ & 5,30 & 1,57 & $-3,80$ \\
i-Amylacetate & $-1,04$ & $-0,31$ & 2,56 & $-1,21$ \\
Hexanoic acid & $-4,41$ & 3,89 & $-1,35$ & 1,88 \\
Total alcohols & 1,38 & $-3,04$ & 4,05 & $-2,39$ \\
Total esters & 0,09 & 0,23 & 0,35 & 0,32 \\
\hline
\end{tabular}

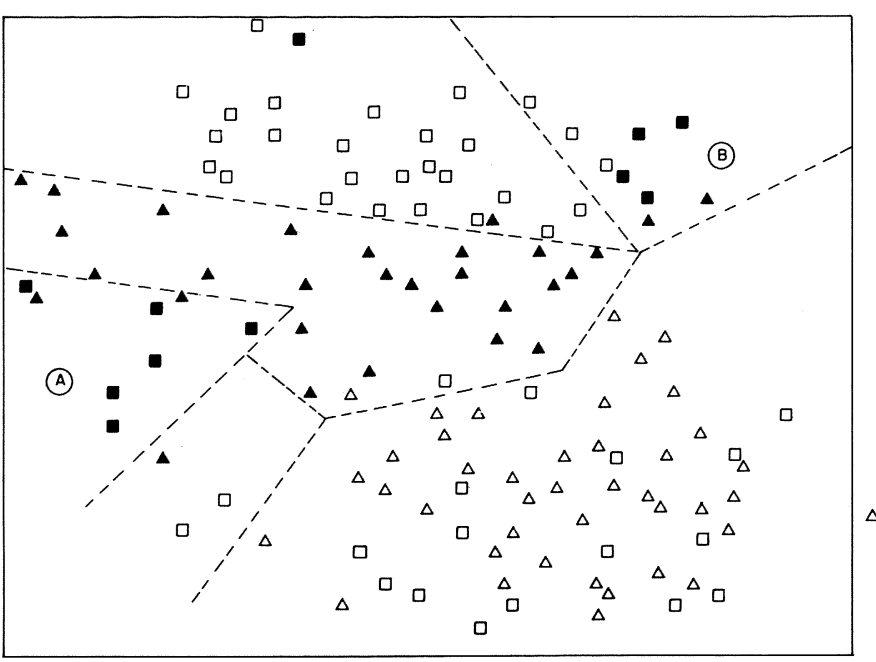

Fig. 4.

Non-linear mapping of 16 dimensional data in two-dimensional space following the selection of 9 original variables and 7 variable ratios.
$\triangle: 0-12$ days TFT (total fermentation time)
口: $13-20$ days TFT
: 21 - 40 days TFT
- $40+$ days TFT
A: wines fermented at $12^{\circ} \mathrm{C}$
B: wines fermented at $20^{\circ} \mathrm{C}$

\section{CONCLUSIONS}

The relative importance of several factors affecting rate of fermentation and wine composition was elucidated in this study. The interpretation of the interactions of several must and fermentation treatments with 20 wine variables has been simplified through cluster analysis and other variable reduction techniques. It is clear that the most effective fermentation accelerator in clear musts is the presence of grape solids. Without sufficient assimilable nitrogen faster fermentations induced in the above manner will lead to wines high in higher alcohols and relatively low in esters. Results obtained in this study suggest that total fermentation times of about 10 days can also produce good quality white wines, provided adequate levels of grape solids and assimilable nitrogen are present.

\section{LITERATURE CITED}

AGENBACH, W. A., 1977. A study of must nitrogen content in relation to incomplete fermentations, yeast production and fermentation activity. Proc. S. Afr. Soc. Enol. Vitic. 66- 68.

ARIES, V., \& KIRSOP, B. H. 1970. Sterol synthesis in relation to growth and fermentation by brewing yeasts inoculated at different concentrations. J. Inst. Brew. 83, $220-223$.

GROAT, MARPE, \& OUGH, C. S., 1978. Effects of insoluble solids added to clarified musts on fermentation rate, wine composition and wine quality. Amer. J. Enol. Vitic. $29112-119$.

HARPER, A. M., DUEWER, D. L., KOWALSKI, B. R. \& FASCHING, J. L., 1977. ARTHUR and experimental data analysis: The heuristic use of a polyalgorithm. "ACS Symposium No. 52" American Chemical Society, Washington D.C. 
JUNGE, C., 1980. Dosage du glycerol dans les vins par la méthode enzymatique. Office international de la vigne et du vin. F. V. 730.

MARAIS, J. \& HOUTMAN, A. C., 1979. Quantative gas chromatographic determination of specific esters and higher alcohols in wine using freon extraction. Amer. J. Enol. Vitic. 30, $250-252$.

SINGLETON, V. L. \& ROSSI, J. A., 1965. Colorimetry of total phenolics with phosphomolybdic phosphotungstic acid reagents. Amer. J. Enol. Vitic. 16, 144 158.
A.O.A.C., 1975. "Official Methods of Analysis." 11 th ed. Association of Official Analytical Chemists, Washington, D.C.

VOS, P. J. A., ZEEMAN, W. \& HEYMANN, HILDEGARDE, 1978. The effect on wine quality of diammonium phosphate additions to musts. Proc. S.A. Soc. Enol. Vitic. 87 - 104.

VOS, P. J. A. \& GRAY, P. S., 1979. The origin and control of hydrogen sulphide during fermentation of grape must. Amer. J. Enol. Vitic. 30, 187 - 197. 